\title{
Hypothermia exerts early neuroprotective effects involving protein conjugation of SUMO-2/3 in a rat model of middle cerebral artery occlusion
}

\author{
GANG LI ${ }^{1,2}$, XIAOZHI LIU ${ }^{3}$, ZHIGUO SU ${ }^{3}$ and DONG ZHANG ${ }^{1}$ \\ ${ }^{1}$ Department of Neurosurgery, Beijing Tiantan Hospital, Capital Medical University, \\ Beijing 100050; ${ }^{2}$ Department of Neurosurgery, Tianjin Anjie Hospital, Tianjin 300409; \\ ${ }^{3}$ Department of Neurosurgery, Tianjin 5th Central Hospital, Tianjin 300450, P.R. China
}

Received April 29, 2016; Accepted March 23, 2017

DOI: $10.3892 / \mathrm{mmr} .2017 .6994$

\begin{abstract}
How hypothermia serves an early protective role against cerebral ischemia remains to be determined. The small ubiquitin-related modifier protein (SUMO) functions as a post-translational modification system and SUMO-2/3 subtypes are often activated in early stress. The present study investigated changes in the protein level of SUMO using western blotting and immunocytochemistry when neurons were exposed to oxygen-glucose deprivation (OGD) in vitro, as well as in a rat model of middle cerebral artery occlusion (MCAO) in vivo. The results demonstrated that a large number of proteins were conjugated to SUMO-2/3 in OGD-injured neurons (within $10 \mathrm{~min}$, peaking at $12 \mathrm{~h}$ ), and was markedly enhanced under conditions of hypothermia $\left(33^{\circ} \mathrm{C}\right)$. Concordantly, lactate dehydrogenase (LDH) release and the apoptosis rate, as determined by LDH and TUNEL assays, respectively, were lower in hypothermia-treated neurons. Similar results were obtained in a rat model of MCAO. Neurological deficit scores were lower in the hypothermia group than in the sham group in the early stage of cerebral ischemia $(\mathrm{P}<0.05)$. However, no significant differences in neurological deficit scores were detected between the hypothermia group and the sham group in the late stage of ischemia (21 days; $\mathrm{P}>0.05$ ). This study implicates a role for SUMO-2/3 in early hypothermia-induced neuroprotection against stroke.
\end{abstract}

Correspondence to: Dr Dong Zhang, Department of Neurosurgery, Beijing Tiantan Hospital, Capital Medical University, 6 TianTan XiLi, Dongcheng, Beijing 100050, P.R. China

E-mail: zhangdong0660@sina.com

Abbreviations: SUMO, small ubiquitin-related modifier; OGD, oxygen-glucose deprivation; MCAO, middle cerebral artery occlusion; $\mathrm{LDH}$, lactate dehydrogenase

Key words: ischemic cerebrovascular disease, hypothermia, neurons, post-translational modification, small ubiquitin-related modifier, neuroprotective effect, middle cerebral artery occlusion
The development of small molecule therapeutics based on SUMO-2/3 may benefit patients with cerebral ischemia.

\section{Introduction}

The annual incidence of stroke in China is 120-180/100,000, with $>2$ million new cases every year (1). At least two-thirds of stroke patients experience varying degrees of physical disability (2). Because of the rapid progression following acute cerebral infarction, there has long been a lack of particularly effective treatments. Intravascular thrombolytic therapy is currently considered to be one of the most promising treatment methods; however, it is only useful in a small number of acute phase patients (3). Therefore, it is important that research continues to develop drugs that can intervene in the progression of ischemia.

Hypothermia therapy is considered to be an effective means for treating cerebral ischemia and hypoxia, high intracranial pressures and other complications that are caused by early stage ischemic cerebrovascular disease (4). Hypothermia is thought to reduce the rate of cerebral metabolism and oxygen consumption, and reduce the synthesis and release of excitotoxic neurotransmitters and inflammatory mediators (5). Despite this, a previous study demonstrated that hypothermia did not reduce the mortality in patients with severe brain damage or massive cerebral infarction (6). This clinical and laboratory discordance sparked renewed discussion on the protection mechanisms of hypothermia, requiring further molecular exploration of hypothermia protection mechanisms.

Small ubiquitin-related modifier (SUMO) functions as a post-translational modification system that maintains protein-protein interactions, cytoplasmic and nuclear translocations, stabilizes the genome and antagonizes ubiquitination (7). SUMO modification is a reversible dynamic equilibrium event, which suggests that SUMO-associated disease may be reversed. For example, the increased conjugation of SUMO with protein is observed during neuroprotection after cerebral ischemia (8). Lee et al (9) found that a large number of proteins had been modified by SUMOs in squirrels during hibernation so as to resist glucose and oxygen 
depletion. Based on previous results, it is hypothesized that the SUMOylation of neuronal proteins may be increased to assist neuroprotection during hypothermia; however, the mechanisms remain unclear.

In the current study, a human cerebral ischemia event was simulated by an in vitro ischemia model that used oxygen-glucose deprivation (OGD), and an in vivo rat model of middle cerebral artery occlusion (MCAO). Results suggest that in early cerebral ischemia, SUMO-2/3, but not SUMO-1, is rapidly activated, and covalently bound to abundant proteins to prevent them from being hydrolyzed by ubiquitin, which greatly increases the tolerance of injured neurons to the hypoxic environment. These findings indicate a novel neuroprotective mechanism of hypothermia. That is, abundant proteins can conjugate to, and be protected by, SUMO-2/3 during early ischemia. These finding may provide an avenue for the development of treatments for patients with cerebral ischemia in the future.

\section{Materials and methods}

Experimental animals. Male Sprague-Dawley (SD) rats $(\mathrm{n}=36$; 12-weeks-old) and 2 female pregnant rats (15-weeks-old) were purchased from the Animal Center of the Cancer Institute of Chinese Academy of Medical Science (Beijing, China), and housed in the Animal Experimental Center, Capital Medical University (Beijing, China), with humidity of $50 \pm 5 \%$ at 20-25 ${ }^{\circ}$. a 12-h light/dark cycle (8:00-20:00) and free access to food and water. All the experiments were performed according to the Principles of Laboratory Animal Care (10) and approved by the Ethics Committee of Capital Medical University (Beijing, China).

Primary neuronal cell cultures. Primary neuronal cell cultures were prepared from the cortex of embryonic rat brains at gestation day 18 . The entire cortex was dissected and digested by $0.125 \%$ trypsin over $10 \mathrm{~min}$. The cells were plated at a density of 100,000 cells $/ \mathrm{cm}^{2}$ in neurobasal medium (Invitrogen; Thermo Fisher Scientific, Inc., Waltham, MA, USA) supplemented with B27, glutamax I, 5\% fetal bovine serum (Invitrogen; Thermo Fisher Scientific, Inc.), and $1 \mu \mathrm{g} / \mathrm{ml}$ gentamicin, at $37^{\circ} \mathrm{C}$ with $5 \% \mathrm{CO}_{2}$. After 3 days in culture, cytosine- $\beta$-D-arabino-furanoside was added to a final concentration of $5 \mu \mathrm{M}$. Cells were then fed twice a week with serum-free neurobasal/B27 medium for an additional 7-9 days.

Immunocytochemistry. The primary neuronal cells were fixed with $4 \%$ paraformaldehyde at room temperature for 10 min, and blocked with 5\% goat serum (Beijing Zhongshan Bio Corp., Beijing, China), $2 \%$ bovine serum albumin (BSA; Beijing Zhongshan Bio Corp.) and $0.1 \%$ Triton-X-100 at $37^{\circ} \mathrm{C}$ for $1 \mathrm{~h}$. Primary antibodies against neuron specific enolase (NSE; 1:1,000 dilution; cat. no. sc-51880; Santa Cruz Biotechnology Inc., Dallas, TX, USA) and glial fibrillary acidic protein (GFAP; 1:4,000 dilution; cat. no. TA500335; Beijing Zhongshan Bio Corp.) were incubated with the cells in moisture box at $4^{\circ} \mathrm{C}$ overnight. The sections were subsequently incubated with goat anti-mouse IgG-CruzFluor ${ }^{\mathrm{TM}} 488$ (1:400 dilution; cat. no. sc-362257; Santa Cruz Biotechnology, Inc.) or goat anti-mouse IgG-CruzFluor ${ }^{\text {TM }} 594$ (1:400 dilution; cat. no. sc-362277; Santa Cruz Biotechnology, Inc.) secondary antibodies at $37^{\circ} \mathrm{C}$ for $1 \mathrm{~h}$, followed by mounting and observation under a fluorescence microscope (Olympus DP 70; Olympus Corporation, Tokyo, Japan).

OGD model and hypothermia treatment. To simulate cerebral ischemia, neuronal cells were cultured in an anoxic chamber (Forma Scientific Anaerobic System). Neurobasal medium free of glucose, L-aspartic acid, L-glutamic acid, and sodium pyruvate was equilibrated overnight in the anoxic chamber with $85 \% \mathrm{~N}_{2}, 10 \% \mathrm{H}_{2}$, and $5 \% \mathrm{CO}_{2}$. At $\sim 90 \%$ confluence, cultures were transferred to the anoxic chamber and washed three times with the anoxic medium. After 10,30 and $60 \mathrm{~min}$, and 2, 4, 8, 12 and $48 \mathrm{~h}$ of OGD exposure, the anoxic medium was replaced with neurobasal/B27 medium and cells were transferred back to the incubator at $37^{\circ} \mathrm{C}$ with a gas mixture of $95 \%$ air and $5 \% \mathrm{CO}_{2}$ for an additional $24 \mathrm{~h}$. For induction of hypothermia, which was performed simultaneously with OGD, the neurobasal/B27 medium was replaced with neurobasal medium pre-cooled to $33^{\circ} \mathrm{C}$ and cultures were placed in a $33^{\circ} \mathrm{C}$ environment.

Western blot analysis. The cells were solubilized in $1 \%$ Nonidet P-40 lysis buffer. Lysates $(40 \mu \mathrm{g})$ were separated by $8 \%$ sodium dodecyl sulfate-acrylamide electrophoresis. Separated proteins were transferred to polyvinylidene fluoride membranes (EMD Millipore, Billerica, MA, USA) and blocking was performed with 5\% skimmed milk at room temperature for $1 \mathrm{~h}$, which was followed by incubation with primary antibodies against SUMO-1 (1:100 dilution; cat. no. sc-130275; Santa Cruz Biotechnology, Inc.) and SUMO-2/3 (1:400 dilution; cat. no. sc-32873; Santa Cruz Biotechnology, Inc.), followed by incubation with horseradish peroxidase (HRP)-conjugated goat anti-mouse IgG secondary antibody (1:2,000 dilution; cat. no. 31430; Thermo Fisher Scientific, Inc.) or goat anti-rabbit IgG secondary antibodies $(1: 5,000$ dilution; cat. no. 31466; Thermo Fisher Scientific, Inc.) at room temperature for $1 \mathrm{~h}$. Conjugated and free SUMO proteins were detected using the same primary antibodies, and distinguished on the same blot based on the different molecular weights of the free and conjugated forms of the proteins. Specific proteins were detected using a SuperSignal protein detection kit (Pierce; Thermo Fisher Scientific, Inc.). The membrane was stripped by Restore ${ }^{\mathrm{TM}}$ Plus Western Blot Stripping Buffer (Invitrogen; Thermo Fisher Scientific, Inc.) and reprobed with a primary monoclonal antibody against $\beta$-actin (1:5,000 dilution; cat. no. MA5-15739; Thermo Fisher Scientific, Inc.) for $2 \mathrm{~h}$ at room temperature. The membrane was washed in TBS- $0.1 \%$ Tween-20 and probed with HRP-conjugated goat anti-mouse IgG secondary antibody (1:2,000 dilution; cat. no. 31430; Thermo Fisher Scientific, Inc.) at room temperature for $1 \mathrm{~h}$. Optical density was measured and analyzed using Quantity One 4.62 software (Bio-Rad Laboratories, Inc., Hercules, CA, USA).

Lactate dehydrogenase ( $L D H)$ activity detection. Samples were divided into control group, OGD group, and OGD + hypothermia group. At $24 \mathrm{~h}$ after experimental conditions were established, LDH content in the conditioned medium was measured by enzyme-linked immunosorbent assay Lactate 
A

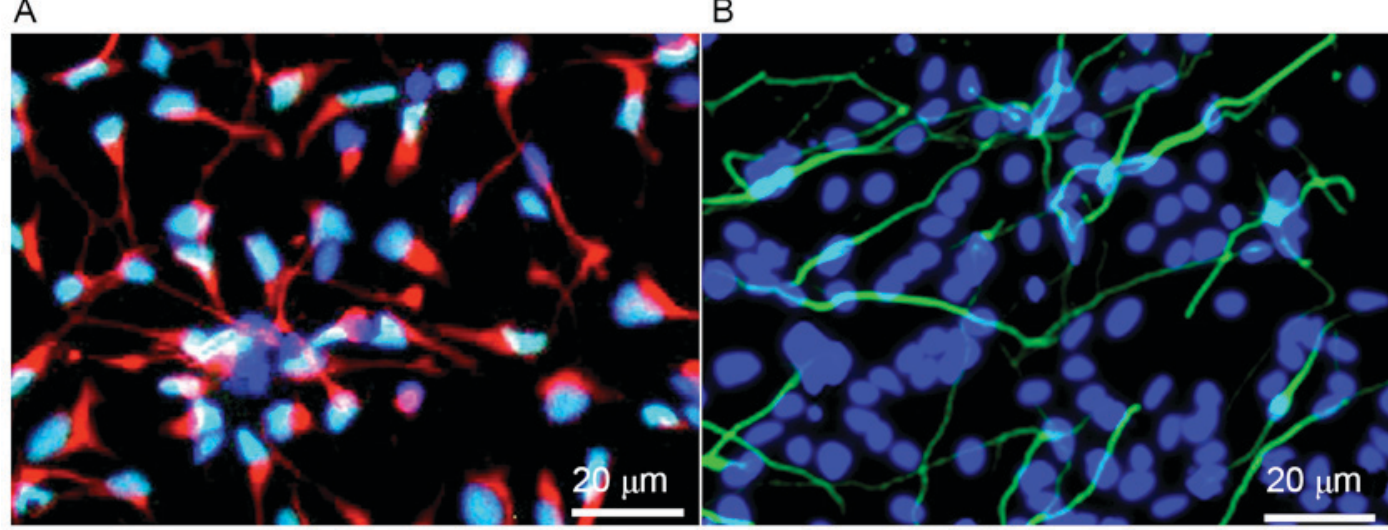

Figure 1. Primary neurons after 10 days in culture. (A) Immunofluorescence staining demonstrated (A) $>90 \%$ of cultured cells expressed neuron-specific enolase protein and (B) $<10 \%$ of cells expressed glial cell specific-marker protein glial fibrillary acidic protein.

Dehydrogenase Assay kit (Colorimetric; cat. no. ab102526; Abcam, Cambridge, UK) in accordance with the manufacturer's instructions.

Apoptosis detection. Neurons were harvested $24 \mathrm{~h}$ after experimental conditions were established, washed with phosphate-buffered saline (PBS), immersed in permeabilization solution $(0.1 \%$ Triton-X-100 $+0.1 \%$ sodium citrate) for $5 \mathrm{~min}$, and incubated with $25 \mu \mathrm{l}$ TUNEL reaction mixture $\left(\right.$ Click-iT ${ }^{\circledR}$ TUNEL Alexa Fluor ${ }^{\circledR} 488$ Imaging assay; Thermo Fisher Scientific, Inc.) in a wet box at $37^{\circ} \mathrm{C}$ for $60 \mathrm{~min}$. After washing with PBS, nuclei were counter-stained with Hoechst 33258. Samples were washed with PBS and deionized water, mounted and observed with a fluorescence microscope. The total number of cells (Hoechst 33258 staining) and the number of apoptotic cells (green fluorescence) were quantified. Apoptotic rate $(\%)$ was equal to the number of apoptotic cells/number of total cells $\mathrm{x} 100$.

Establishment of a rat model of MCAO and hypothermia treatment. Adult SD rats $(\mathrm{n}=48)$ were randomly divided into three groups, with 16 rats in each group. The experiment was performed using a small animal ventilator. Body temperature was monitored with a rectal thermometer. A 1-cm-long medial longitudinal incision was made from the manubrium to mandible. The left common carotid artery, external carotid artery, and internal carotid artery were isolated under a microscope. The distal segment of the common carotid artery and the proximal segment of the external carotid artery were ligated. A nylon suture with a 0.23 -mm-diameter tip and $0.18-\mathrm{mm}$ trunk was inserted into the middle cerebral artery through the common carotid artery $(\sim 12 \mathrm{~mm})$ and fixed. In the sham surgery group (control group), the arteries were exposed, but without the insertion of a nylon suture. In the hypothermia group, the experiment was carried out on controlled temperature blankets. After anesthesia, SD rat body temperature was controlled between 32 and $34^{\circ} \mathrm{C}$ with a rectal thermometer. Following occlusion, body temperature was allowed to gradually recover to normal over $10 \mathrm{~h}$. Laboratory animals were housed in a quiet clean room. At 1, 7, 14 and 21 day after the models were established, the neurological deficit of all rats was evaluated as previously described (11).
Immunofluorescence analysis of SUMO proteins. At 4 days after model establishment, 4 rats in each group were sacrificed, and the brain tissue was isolated and fixed with $10 \%$ formaldehyde at $4^{\circ} \mathrm{C}$ for $24 \mathrm{~h}$, and subsequently embedded in paraffin and sliced into $5-\mu \mathrm{m}$-thick coronal sections. Hippocampal sections were de-waxed with xylene and alcohol, and hydrated through an alcohol gradient. Following antigen retrieval with $0.01 \mathrm{M}$ citric acid buffer $(\mathrm{pH}=6.0)$, sections were blocked with $5 \%$ goat serum at room temperature for $10 \mathrm{~min}$ and incubated with mouse anti-SUMO-1 (1:100 dilution; cat. no. sc-5308; Santa Cruz Biotechnology, Inc.) and rabbit anti-SUMO-2/3 (1:500 dilution; cat. no. sc-32873; Santa Cruz Biotechnology, Inc.) primary antibodies at $4^{\circ} \mathrm{C}$ overnight. The samples were washed repeatedly, incubated with goat anti-mouse IgG-CruzFluor $^{\mathrm{TM}} 488$ (1:400 dilution; cat. no. sc-362257; Santa Cruz Biotechnology, Inc.) or goat anti-rabbit IgGCruzFluor $^{\text {TM }} 594$ (1:400 dilution; cat. no. sc-362282; Santa Cruz Biotechnology, Inc.) secondary antibodies at $37^{\circ} \mathrm{C}$ for $1 \mathrm{~h}$, counter-stained with DAPI, mounted with fluorescence mounting medium and observed using a fluorescence microscope.

Statistical analysis. Data are expressed as the mean \pm standard deviation. Data were analyzed using one-way analysis of variance by SPSS 13.0 software (SPSS Inc., Chicago, IL, USA). The Tukey test was performed for multiple comparisons. $\mathrm{P}<0.05$ was considered to indicate a statistically significant difference.

\section{Results}

Neuronal culture. Immunofluorescence demonstrated that after 10 days of primary culture, $>90 \%$ of the primary cells expressed neuron-specific protein NSE, and $<10 \%$ of cells expressed the glial cell specific marker proteins, GFAP (Fig. 1). These results indicated that neurons were successfully cultured at a high purity.

Western blot analysis. SUMO-1 and SUMO-2/3 expression was identified in the early stage $(48 \mathrm{~h})$ of OGD. Results revealed that free and conjugated SUMO-1 expression did not change within $48 \mathrm{~h}$ of OGD, whereas conjugated SUMO-2/3 
Table I. Neurological impairment scores in rats.

Time after MCAO (days)

\begin{tabular}{lcccc}
\cline { 2 - 5 } Group & 1 & 7 & 14 & 21 \\
\hline Control & $0.14 \pm 0.03$ & $0.11 \pm 0.03$ & $0.08 \pm 0.02$ & $0.09 \pm 0.02$ \\
MCAO & $33.12 \pm 3.26^{\mathrm{a}}$ & $23.15 \pm 4.56^{\mathrm{a}}$ & $16.24 \pm 3.18^{\mathrm{a}}$ & $12.31 \pm 2.51^{\mathrm{a}}$ \\
MCAO + hypothermia & $32.34 \pm 3.09^{\mathrm{a}}$ & $19.71 \pm 3.68^{\mathrm{a}, \mathrm{b}}$ & $12.73 \pm 3.17^{\mathrm{a}, \mathrm{b}}$ & $11.72 \pm 2.46^{\mathrm{a}}$
\end{tabular}

${ }^{\mathrm{a}} \mathrm{P}<0.05$ vs. control group; ${ }^{\mathrm{b}} \mathrm{P}<0.05$ vs. $\mathrm{MCAO}$ group. MCAO, middle cerebral artery occlusion.
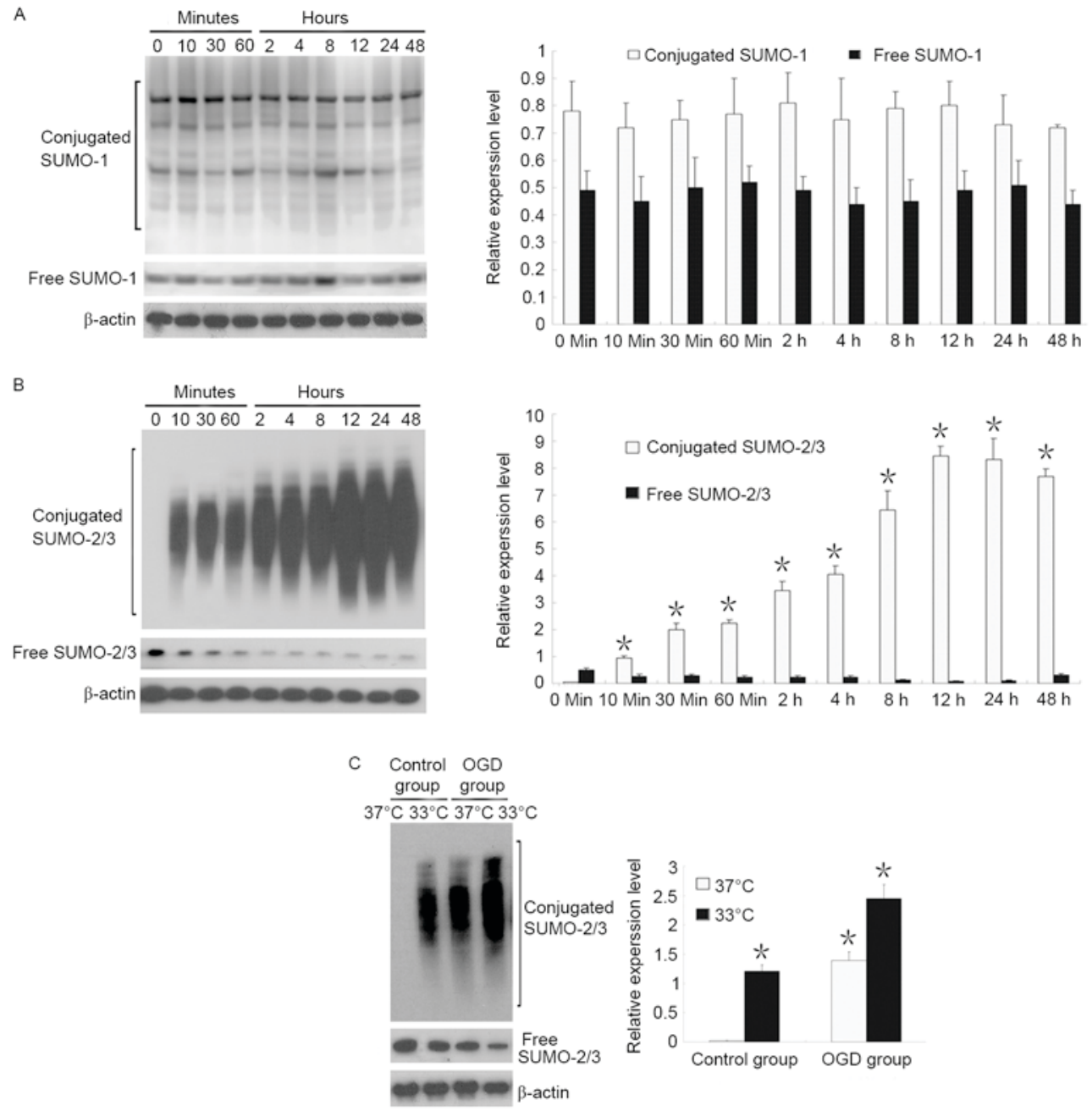

Figure 2. Effects of OGD and hypothermia on SUMO-1 and SUMO-2/3 expression in neurons. (A) After $48 \mathrm{~h}$ of OGD, SUMO-1 did not change with time. (B) An immediate and prolonged increase in SUMO-2/3 was observed after OGD. "P<0.05 vs. 0 min conjugated SUMO-2/3. (C) Hypothermia induced a further increase in the conjugation of proteins with SUMO-2/3. ${ }^{*} \mathrm{P}<0.05$ vs. $37^{\circ} \mathrm{C}$ control group. SUMO, small ubiquitin-related modifier; OGD, oxygen-glucose deprivation.

expression increased starting from $10 \mathrm{~min}$ of OGD and reached a peak at $12 \mathrm{~h}$ of $\mathrm{OGD}$, and a gradual decrease in free SUMO-2/3 (Fig. 2A and B).

The effects of temperature on SUMO- 1 and SUMO-2/3 expression were also observed. Results demonstrated that when neurons were cultured at a normal temperature $\left(37^{\circ} \mathrm{C}\right)$, there was almost no conjugated SUMO-2/3, and that SUMO-2/3 was present only in an unconjugated state. When neurons were cultured in hypothermia conditions $\left(33^{\circ} \mathrm{C}\right)$, conjugated
SUMO-2/3 expression markedly increased, suggesting that abundant proteins were bound to SUMO-2/3. Under OGD conditions, hypothermia induced a 1.8 -fold increase in conjugated SUMO-2/3 expression compared with OGD at $37^{\circ} \mathrm{C}$ (Fig. 2C).

Neuroprotective effect of hypothermia on OGD-induced injury. An apoptosis assay demonstrated that apoptosis was increased by ODG, and this effect of apoptosis was reduced by 


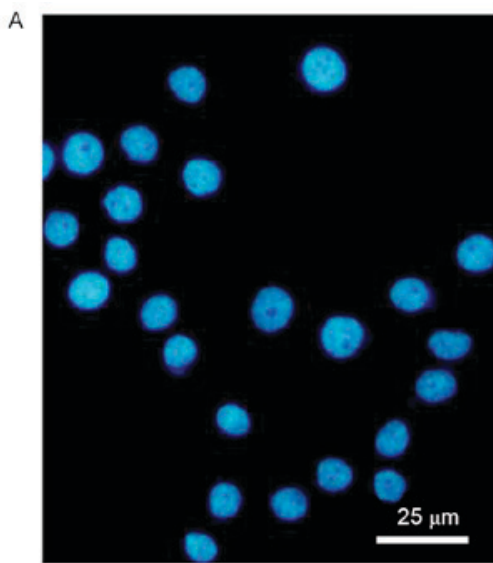

Control group

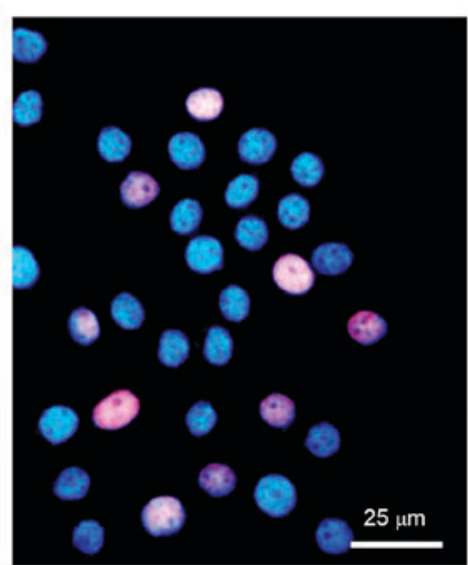

OGD group

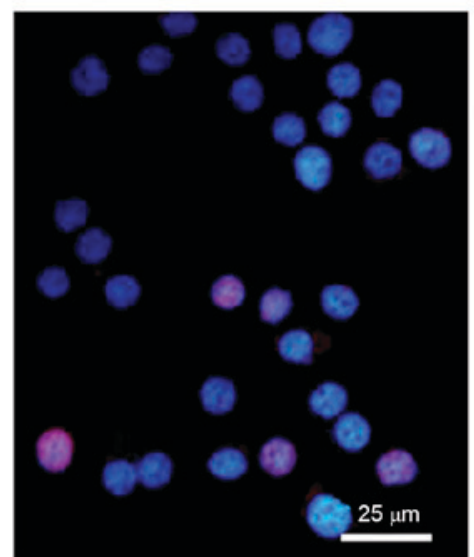

OGD+hypothermia group
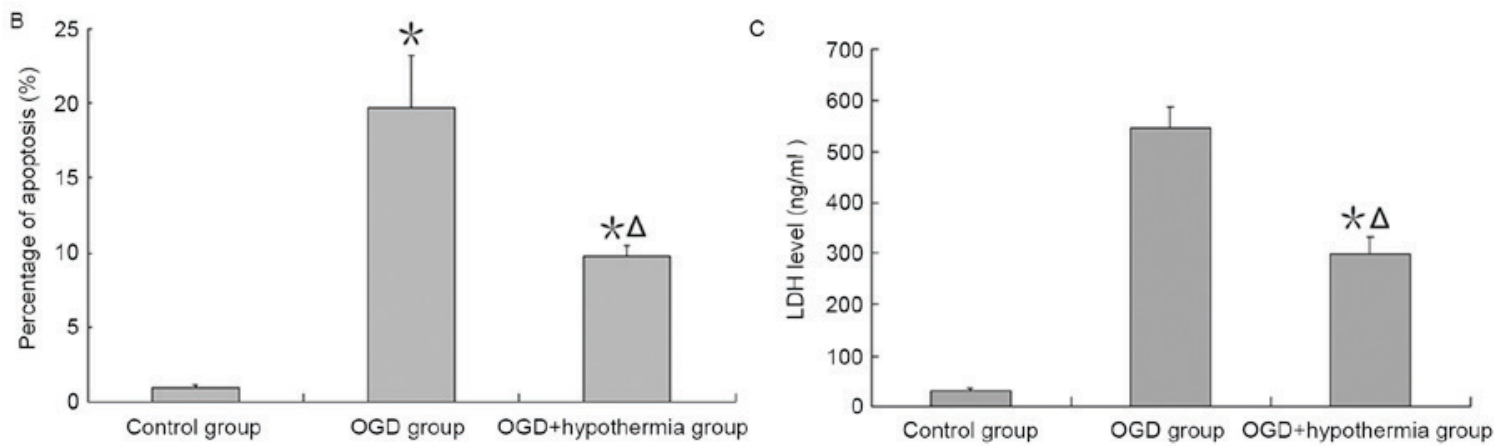

Figure 3. Protective effects of hypothermia on OGD-injured neurons. (A) Representative images of in situ apoptosis detection and (B) the percentage of apoptotic cells. (C) Enzyme-linked immunosorbent assay for $\mathrm{LDH}$ expression. ${ }^{*} \mathrm{P}<0.05$ vs. control group; ${ }^{\Delta} \mathrm{P}<0.05$ vs. OGD group. OGD, oxygen-glucose deprivation; LDH, lactate dehydrogenase.
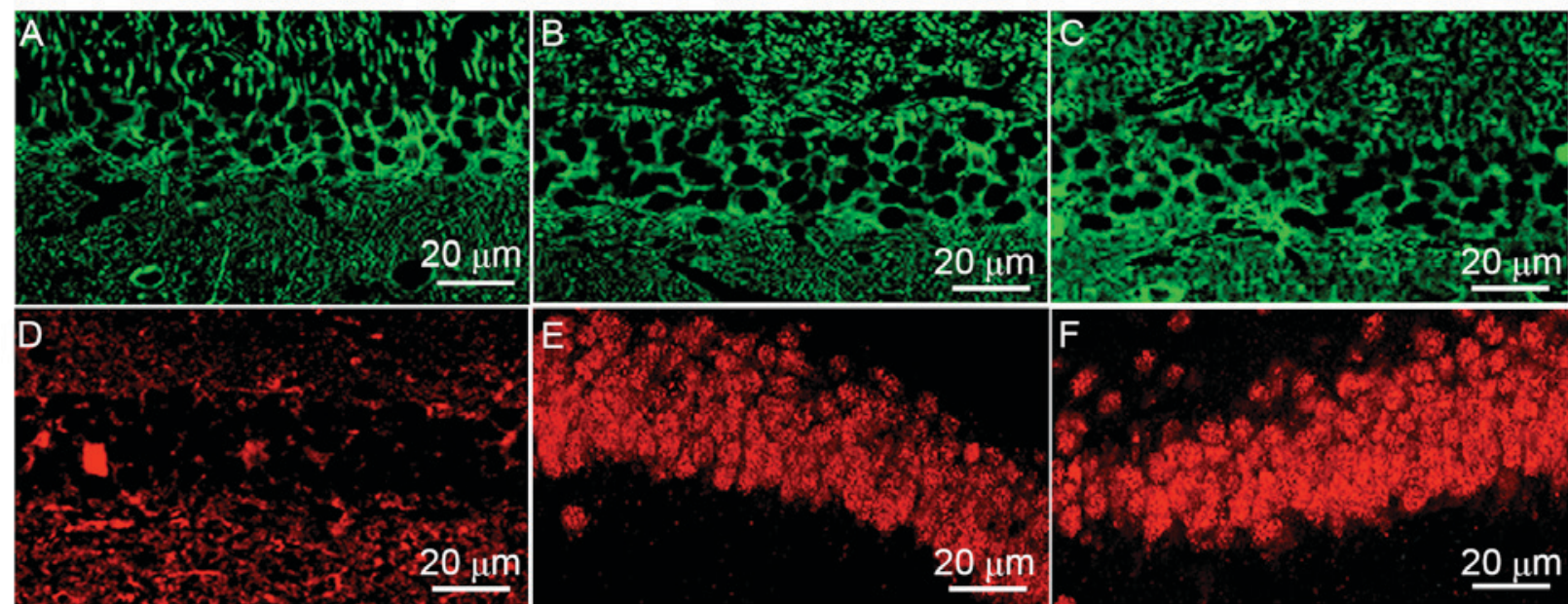

Figure 4. Immunofluorescence analysis of SUMO protein expression in rat hippocampal sections. The expression of SUMO-1 in (A) control group, (B) MCAO group and (C) MCAO + hypothermia group rats. The expression of SUMO-2/3 in (D) control group, (E) MCAO group and (F) MCAO + hypothermia group rats. SUMO, small ubiquitin-related modifier; MCAO, middle cerebral artery occlusion.

hypothermia (Fig. 3A and B). Additionally, the results revealed that LDH expression was very low in neurons cultured in normal conditions, but high when neurons were exposed to OGD conditions. LDH expression was significantly lower in OGD-injured neurons in the hypothermia group than in the ODG normal temperature group ( $F=38.4, \mathrm{P}<0.05$; Fig. $3 \mathrm{C})$.
Neurological deficit scores. Compared with the control group, neurological deficit scores were higher in the MCAO group at the same time point. Neurological deficit scores in the $\mathrm{MCAO}+$ hypothermia group were lower than in the MCAO group in the early stage of cerebral ischemia, but not in the late stage of ischemia (>21 days; Table I). 
SUMO-2/3 transport from cytoplasm to nucleus. The localization of SUMO-1 (Fig. 4A-C) and SUMO-2/3 (Fig. 4D-F) in rat hippocampal neurons differed according to the treatment group. Results revealed that SUMO-1 was mainly located in the cytoplasm in the control group. MCAO and hypothermia did not impact on SUMO-1 expression or localization. However, MCAO induced SUMO-2/3 translocation from the cytoplasm to the nucleus, and hypothermia enhanced this transport.

\section{Discussion}

Massive cerebral infarction has a high mortality and disability rate due to abrupt onset and rapid progression. Hypothermia has been considered as one of the early and effective treatment methods for cerebral infarction-induced severe ischemia and hypoxia. A recent large-scale clinical investigation suggested that hypothermia does not significantly reduce the mortality in patients with severe brain damage or massive cerebral infarction, but may assist rescuing ischemic penumbra neurons (12). The current understanding of hypothermia as a treatment encompasses the following (13-17): i) Hypothermia decreases the cellular metabolism rate and increases energy reserve for recovery; ii) hypothermia reduces the accumulation of inflammatory substances in the local region, and improves the local microenvironment; and iii) hypothermia enhances the tolerance of injured nerve cells to the microenvironment by reducing the activity of proteolytic enzymes. Based on the above, it seems feasible to use targeted hypothermia neuroprotection therapies in the early, critical window.

The biological functions of SUMO include maintaining genomic stability, regulating protein interactions, adjusting cytoplasmic protein transport and localization, controlling the activity of transcription factors and antagonizing ubiquitination (18-22). SUMO modification is a reversible dynamic process, which indicates that SUMOylation-associated disease can be reversed. Yang et al $(23,24)$ reported that cerebral ischemia activates a SUMO-2/3-mediated molecular mechanism pathway and exert a neuroprotective effect. A previous study demonstrated that following ischemic cerebrovascular disease, proteins that are strongly associated with oxidative stress, inflammation, DNA synthesis, energy transfer and metabolism via SUMO can remove foreign substances, resist inflammation and balance cell proliferation and apoptosis (25). In 2011, Lee et al (9) reported that the SUMO modification of a large number of proteins increased in squirrels during hibernation, presumably to resist glucose and oxygen depletion. Thus, we hypothesized that SUMOs may have an important role in the molecular mechanisms of hypothermia-mediated neuroprotection.

In the present study, we used an in vitro OGD neuronal model that incorporated a hypothermia therapeutic element. SUMO-2/3 was activated in neurons under ischemic and hypoxic conditions, corresponding to increasing conjugated SUMO-2/3 protein expression. This result suggests that a certain amount of SUMO-2/3 is reserved in neurons for use during stress responses, yet the quantity of SUMO-2/3 in cells is ultimately limited. The amount of SUMO-2/3 appeared to reach a peak at $12 \mathrm{~h}$ after the onset of stress. After this time, more SUMO-2/3 would presumably need to be synthesized to supplement the lost SUMO-2/3.

Subsequent experimental results found that hypothermia could not only increase the expression level of conjugated SUMO-2/3 in normal neurons, but also enhance the conjugation of SUMO-2/3 to target proteins in OGD-injured neurons. These findings indicate that hypothermia could initially increase the conjugation of proteins to SUMO-2/3 in a physical capacity, and may also enhance the conjugation of SUMO-2/3 to target proteins via intervention from other stress factors.

To verify that SUMO-2/3 activation under hypothermia is a neuroprotective response, the degree of apoptosis of OGD-injured neurons cultured in vitro was investigated. Hypothermia decreased the degree of apoptosis in early OGD. In the MCAO model, hypothermia reduced rat neurological deficits in the early stage, but this effect diminished with time. Ultimately, there was no statistical difference between the neurological deficit score of the MACO group and the $\mathrm{MCAO}+$ hypothermia group at days 21 . Previous findings indicate that hypothermia may delay the apoptosis of injured cells in the early stage, but could not ultimately stop this process, and may even result in the acceleration of apoptosis upon rewarming (26). Our evidence supports this theory. The precise mechanism of how this occurs requires further investigation. Part of this may relate to the localization of SUMO, where our in vivo results suggest that hypothermia increases SUMO-2/3 transport from the cytoplasm to the nucleus, involving increased conjugation with target protein. Future studies may be able to determine whether the enhanced SUMO-2/3 conjugation is only a temporary feature induced by hypothermia, or a genuine phenomenon involving DNA repairing leading to neuron regeneration.

In conclusion, the current study confirmed that hypothermia may enhance the conjugation of SUMO-2/3 to target proteins and halt cellular apoptosis in early stage of ischemia. This result serves to enrich the understanding of the molecular mechanism of brain protection in hypothermia. The development of small molecule drugs based on SUMO-2/3 may provide a putative therapeutic target for patients with cerebral ischemia. Ultimately, improving the understanding of the hypothermia process will lead to more effective protocols for use in the critical, early stage of ischemia therapy.

\section{Acknowledgements}

The present study was supported by the China National Natural Scientific Fund (grant nos. 81471175 and 81371292).

\section{References}

1. Tu Y, Chen C, Sun HT, Cheng SX, Liu XZ, Qu Y, Li XH and Zhang S: Combination of temperature-sensitive stem cells and mild hypothermia: A new potential therapy for severe traumatic brain injury. J Neurotrauma 29: 2393-2403, 2012.

2. Kirton A and Deveber G: Life after perinatal stroke. Stroke 44: 3265-3271, 2013

3. Wu S, Sena E, Egan K, Macleod M and Mead G: Edaravone improves functional and structural outcomes in animal models of focal cerebralischemia: A systematic review. Int J Stroke 9: 101-106, 2014.

4. Wu TC and Grotta JC: Hypothermia for acute ischaemic stroke. Lancet Neurol 12: 275-284, 2013. 
5. Zhang M, Wang H, Zhao J, Chen C, Leak RK, Xu Y, Vosler P, Chen J, Gao Y and Zhang F: Drug-induced hypothermia in stroke models: Does it always protect? CNS Neurol Disord Drug Targets 12: 371-380, 2013.

6. Kallmünzer B, Kollmar R and Schwab S: Therapeutic hypothermia in acute brain injury. Nervenarzt 83: 975-981, 2012.

7. Droescher M, Chaugule VK and Pichler A: SUMO rules: Regulatory concepts and their implication in neurologic functions. Neuromolecular Med 15: 639-660, 2013.

8. Cubeñas-Potts C and Matunis MJ: SUMO: A multifaceted modifier of chromatin structure and function. Dev Cell 24: 1-12, 2013

9. Lee YJ, Mou Y, Maric D, Klimanis D, Auh S and Hallenbeck JM Elevated global SUMOylation in Ubc9 transgenic mice protects their brains against focal cerebral ischemic damage. PLoS One 6 e25852, 2011.

10. Guide for the care and use of laboratory animals. Public Health Service, National Institute of Health: NIH Publication No. 86-23. Revised 1985.

11. Reglodi D, Tamás A and Lengvári I: Examination of sensorimotor performance following middle cerebral artery occlusion in rats. Brain Res Bull 59: 459-466, 2003.

12. Meixensberger J and Renner C: Therapeutic hypothermia in the intensive care unit. Anaesthesist 56: 945-948, 2007 (In German).

13. Jordan JD and Carhuapoma JR: Hypothermia: Comparing technology. J Neurol Sci 261: 35-38, 2007.

14. Ramani R: Hypothermia for brain protection and resuscitation. Curr Opin Anaesthesiol 19: 487-491, 2006.

15. Alzaga AG, Cerdan M and Varon J: Therapeutic hypothermia. Resuscitation 70: 369-380, 2006

16. Nogoy N: Neuroproteomics: The hunt for biomarkers of neurotrauma. Andrew ottens talks to nicole nogoy. Expert Rev Proteomics 4: 343-345, 2007.
17. Han HS, Qiao Y, Karabiyikoglu M, Giffard RG and Yenari MA: Influence of mild hypothermia on inducible nitric oxide synthase expression and reactive nitrogen production in experimental stroke and inflammation. J Neurosci 22: 3921-3928, 2002.

18. Guo C and Henley JM: Wrestling with stress: Roles of protein SUMOylation and deSUMOylation in cell stress response. IUBMB Life 66: 71-77, 2014.

19. Feligioni M and Nisticò R: SUMO: A (oxidative) stressed protein. Neuromolecular Med 15: 707-719, 2013.

20. Sriramachandran AM and Dohmen RJ: SUMO-targeted ubiquitin ligases. Biochim Biophys Acta 1843: 75-85, 2014.

21. Huang CJ, Wu D, Khan FA and Huo LJ: DeSUMOylation: An important therapeutic target and protein regulatory event. DNA Cell Biol 34: 652-660, 2015.

22. Abeywardana T and Pratt MR: Extent of inhibition of $\alpha$-synuclein aggregation in vitro by SUMOylation is conjugation site- and SUMOisoform-selective. Biochemistry 54: 959-961, 2015.

23. Yang W, Thompson JW, Wang Z, Wang L, Sheng H, Foster MW, Moseley MA and Paschen W: Analysis of oxygen/glucose-deprivation-induced changes in SUMO3 conjugation using SILAC-based quantitative proteomics. J Proteome Res 11: 1108-1117, 2012.

24. Yang W, Wang L and Paschen W: Development of a high-throughput screening assay for inhibitors of small ubiquitin-like modifier proteases. J Biomol Screen 18: 621-628, 2013.

25. Yang W, Sheng H, Homi HM, Warner DS and Paschen W: Cerebral ischemia/stroke and small ubiquitin-like modifier (SUMO) conjugation-a new target for therapeutic intervention? J Neurochem 106: 989-999, 2008.

26. Olsen TS, Weber UJ and Kammersgaard LP: Therapeutic hypothermia for acute stroke. Lancet Neurol 2: 410-416, 2003. 